\title{
Clinical Competency: Simulation Compared with Lectures in Training Registered General Nursing (RGN) at Time of Completion of Program-A Systematic Review
}

\author{
Tulukuu Perekuu1 ${ }^{1}$ Ba-Etilayoo Atinga ${ }^{2}$, Albert Henyo ${ }^{2}$, Gilbert Graham Addah ${ }^{2}$ \\ ${ }^{1}$ Holy Family Nursing and Midwivery Training College, Berekum, Ghana \\ ${ }^{2}$ Department of Nursing, University of Energy and Natural Resources, Sunyani, Ghana \\ Email: tperekuu@gmail.com, ba-etilayoo.atinga@uenr.edu.gh, albert.henyo@uenr.edu.gh,nhyirabagraham@gmail.com
}

How to cite this paper: Perekuu, T., Atinga, B.-E., Henyo, A. and Addah, G.G. (2022) Clinical Competency: Simulation Compared with Lectures in Training Registered General Nursing (RGN) at Time of Completion of Program-A Systematic Review. Open Journal of Nursing, 12, 60-69. https://doi.org/10.4236/ojn.2022.121005

Received: December 1, 2021

Accepted: January 25, 2022

Published: January 28, 2022

Copyright () 2022 by author(s) and Scientific Research Publishing Inc. This work is licensed under the Creative Commons Attribution International License (CC BY 4.0).

http://creativecommons.org/licenses/by/4.0/ (c) (i) Open Access

\begin{abstract}
Background: Advancing the science of nursing education through technology into teaching and learning is essential to prepare nurses for the changing healthcare environment. Objective: To synthesise evidence in nursing students' competency in the clinical setting immediately after completion of training with simulation compared with lectures. Methods: Databases were sourced electronically from CINAHL and PubMed using keywords Simulation, lecture, clinical competency and new graduates. Study Eligibility Criteria, Participants and Interventions: Published articles addressing the research objectives, written in English, spaning between September 2010 and March 2019 directly answer the PSCOT question with results applicable to the population specific-nursing students, simulation was utilized. Study Appraisal and Synthesis Methods: Extracted data from sorted studies were analysed and assessed for quality using the John Hopkins Evidence-Based Appraisal Tools. Results: The two-database yielded 520 articles, 10 met the eligibility criteria. Findings documented three important themes: 1) Clinical competence among nurses; 2) Medical residents' clinical competence and 3) Was about the competence of anaesthesiology residents. Limitations: The identified studies used small sample sizes ranging from 20 to 98 participants in the single research studies. Conclusions: The review of the literature found that the active teaching strategy of simulation was more effective in promoting clinical competency in nursing students compared with the lecture method. The evidence-based teaching project proposes the use of simulation as the primary teaching method
\end{abstract}


to improve the clinical competency abilities of registered general nursing (RGN) diploma students.

\section{Keywords}

Clinical, Competency, Simulation, Lecture, Registered, General, Nursing

\section{Introduction}

\subsection{Rationale}

Traditionally, Ghanaian nursing students receive explicit academic instructions in the classroom setting and acquire a wide variety of nursing skills in a clinical setting during their clinical practicum. Due to a large number of certificates, diplomas and undergraduate nursing programs coupled with the competition over clinical settings, nursing education faces several challenges. These challenges include greater demand for clinical placements that leads to fewer opportunities for nursing students to obtain chances to take part in actual patient care [1]; overcrowding of teaching hospitals with medical and nursing students from many different academic institutions. All these impede the overall clinical education process [1]. The challenges pose a serious threat to the development of clinical competencies among the college registered general nursing diploma students. Christmals et al. (2018) [2] reported a connection between students' inexperience and their ability to competently carry out vital tasks, in the clinical setting. Thus, transforming nursing education to enhance patient safety mandates the advent of standardized approaches and advanced technologies to reproduce the day-to-day situations found in actual healthcare settings.

The theory-practice gap creates a huge problem for educational institutions in making sure that graduates are ready to provide the best healthcare possible for an ever more at-risk population [3]. The gap can be aggravated by reliance on traditional teaching methods and fewer clinical opportunities for nursing students to practice nursing skills [1]. Therefore, nurse educators must address the limited experience and develop or adopt other methods for teaching clinical skills to help in bridging the gap; thus, increasing clinical competence. The project explored the effectiveness of two instructional methods, Simulation and traditional teaching method (lecture), in a College in Ghana which offers diploma nursing education to determine the effectiveness of clinical competence in inpatient care settings.

Simulation-based clinical education in nursing refers to a variety of activities using patient simulators, including devices, trained persons, lifelike virtual environments, and role-playing, not just handling mannequins. With realistic clinical scenarios, simulation-based educational interventions in nursing can train novice as well as experienced nurses, helping them develop effective non-technical skills, practice rare emergency situations, and providing a variety of authentic 
life-threatening situations.

Concerning internal evidence, there has been increasing complaints in recent about newly qualified nurses from the college who are insufficiently prepared to fully participate in inpatient care. The faculty or instructors at the college attributed this lack of clinical competence of the new graduate from the college to increase the number of students at the various clinical sites. That was verbally confirmed by the Principal or head of the College. The report has prompted a reexamination of the primary teaching method for the practical aspect of nursing to improve clinical competency upon completion of the program. Like most of the didactic nursing courses at the college, the primary teaching method is lecture, and this has driven a need to incorporate more active teaching methods to improve clinical competency in students. The active teaching strategy the project offers to address is simulation.

An institutional vision and mission statements are unspoken contracts between the organization and various stakeholders. A well-written statement can guide decision-making, resource allocations, policy decisions, and how the school operates. By making its purpose clear, colleges can put their goals and objectives into action [4].

The aim of the college where this project took place is to train the requisite and qualified Nursing and Midwifery Personnel to provide the manpower needs of health institutions in the country and to uphold ethical and professional standards. As a Catholic Institution, the College instils in its Nursing and Midwifery students the Gospel Values of the Kingdom of God. These are love, compassion, forgiveness, humility, sacrifice, tolerance; and care for the sick, women, children, and the vulnerable.

The Vision of the College is to continue the Christ Healing Ministry, by training young men and women to provide high-quality patient care through professional nurses and midwives with ethical and moral standards; who are conscientious as well as professionally competent, motivated and united in their common respect for fundamental human values. The college also has its mission, to train all respective students without regard to religion, ethnicity, nationality and social standings as nurses and midwives to render nursing and midwifery service to the people of Ghana and beyond.

Lack or low clinical competence of the new graduates from the college is a deviation of the vision of the college hence the need to gather evidence to support a new evidence-based teaching strategy, that will help enhance the clinical competency level of new graduates from the college to help bridge the theory-practice gap.

\subsection{Objective}

To synthesise evidence in nursing students' competency in the clinical setting immediately after completion of training with simulation compared with a lecture. We believe that no previous study has summarised the literature on this topic. 


\subsection{Research Question}

Using a PSCOT format, the question for the project is: In the registered general nursing (RGN) students $(\mathrm{P})$ how does simulation (S) compared with a lecture (C) affect clinical competency? (O) after the program (T).

\section{Methods}

A systematic review was done per the Preferred Reporting Items for Systematic Reviews and Meta-Analyses (PRISMA) guidelines (Figure 1).

\section{PRISMA 2009 Flow Diagram}

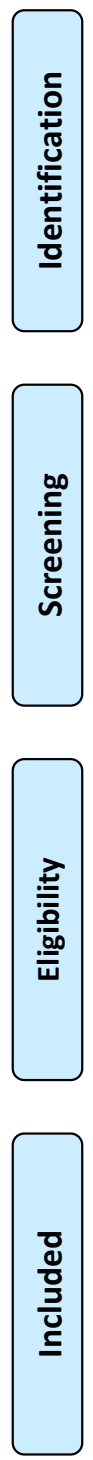

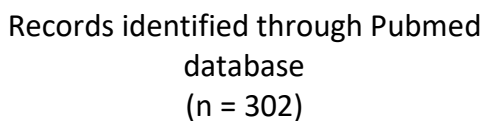
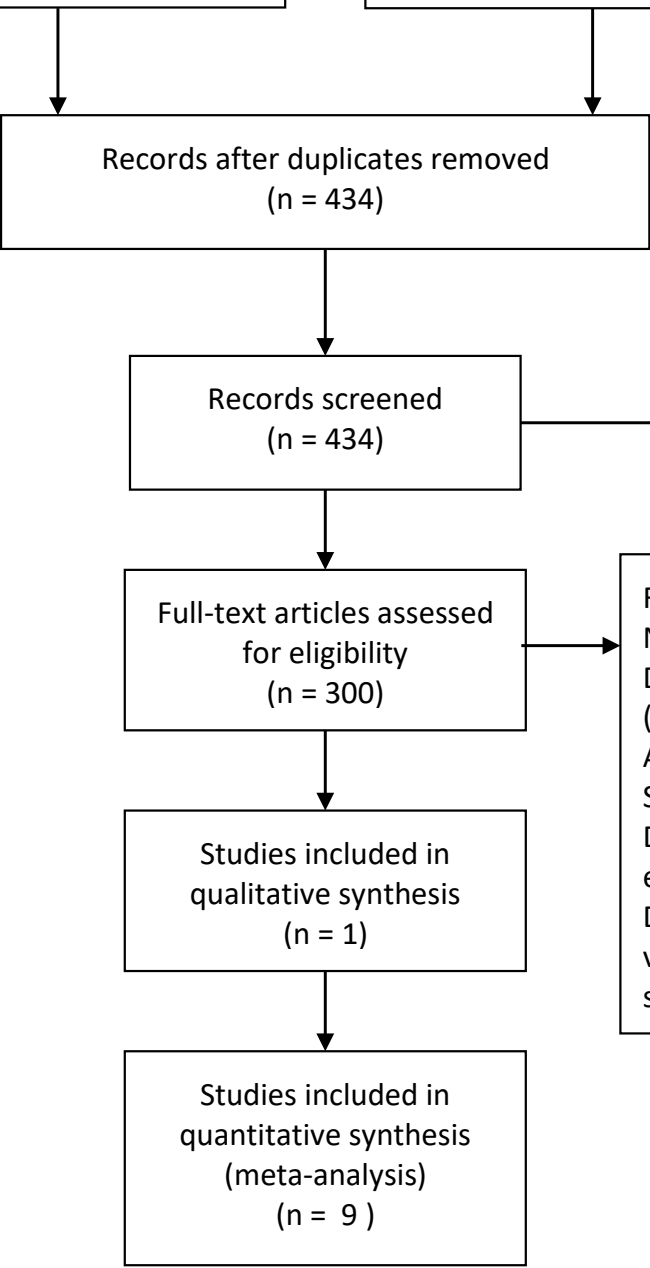

Additional records identified through CINAHL database $(n=218)$

Figure 1. PRISMA flowchart detailing search results and the selection of studies. 


\subsection{Eligibility Criteria}

Peer-reviewed article evidence that directly answers PSCOT question, written in English, published between September 2010 and March 2019, and the evidence results applicable to the population specifics-nursing students, simulation. The review excluded evidence of populations that were not student nurses studies not answering the PSCOT question or using non-valid and unreliable methods to address the question, and evidence which results does not apply to the population.

\subsection{Information Sources and Search}

Evidence from two electronic databases was searched: CINAHL and PubMed. Multiple keywords were used to search the above databases: clinical competence(s), nursing student(s), simulation, theory-practice gap(s), lecture, traditional lecture(s), didactic lecture(s), and simulation compare with a lecture. The keywords were combined using "OR" then combined using "AND". A detailed example of the search strategy for evidence employed.

\subsection{Study Selection}

All peer-reviewed articles that met the eligibility criteria were selected. Search lists were counter screened by the authors (TP, BA) to avoid duplication. Evidence that fell short of the eligibility criteria were excluded before reviewing the full text. In cases of uncertainties, an additional person was consulted to resolve it by referring back to the protocol. Evidence was evaluated for quality and then included.

\subsection{Data Extraction}

Regarding earlier systematic reviews done, a form was developed to gather evidence. Evidence gathered from the identified articles for this systematic review included: country and setting, details of participants, objectives of the study, research design, description of the main findings related to the three research questions, and the reported limitations of each study.

\subsection{Data Synthesis}

The extracted evidence was critically analysed to make meaning of the narrative description of the findings. Thematic synthesis was employed for both the qualitative and quantitative research outcomes. The extracted data were categorised into level type, overall quality rating and synthesis findings that answer the evidence-based practice in question. After reviewing for relevance and appropriateness to the topic, papers that did not discuss simulation or lecture explicitly were excluded.

\subsection{Quality Assessment}

The assessment of the selected studies was done by both authors (PT, BA) using 
a PSCOT format, the question for the project is: In the registered general nursing (RGN) students (P) how does simulation (S) compared with a lecture (C) affect clinical competency? $(\mathrm{O})$ after the program $(\mathrm{T})$. The studies were screened for clarity relative to the research question, and whether the collected data addressed the research questions. Studies that met the screening process were then appraised about the study design. Studies that met all assessment criteria scored 1 ; studies that met fewer criteria scored less than 1 .

\section{Results}

\subsection{Study Selection}

The total search of literature from the two databases yielded 520 published articles. After reviewing for relevance and appropriateness to the topic, papers that did not discuss simulation or lecture explicitly were excluded. A total of 10 articles were identified as eligible for inclusion in this review, based on contributions to the existing body of knowledge in terms of the methodological approaches, the target populations, the type and the frequency of the clinical competence, reporting of clinical competence, and the interventions utilized to increase clinical competence among nursing students. Of the 10 studies, seven were conducted in the United States, one in Iran, and the remaining three were systematic reviews. The majority of the studies [5] were concerned with clinical competence among nurses; with two [2] studies concerning medical residents' clinical competence and one [1] was about the competence of anaesthesiology residents. Consequently, 10 studies were included in the final review.

\subsection{Narrative of Evidence Synthesis}

\subsubsection{Population (P)}

Half [5] of the studies considered in the literature used nursing students as the population of the study. The synthesis of literature supported the use of simulation for assisting nursing students to feel more confident in performing clinical work. The use of simulation supported the curriculum and enhanced the knowledge level and learning of each student as the faculty continued to use simulation throughout the nursing courses and also nursing students, participation in the simulation was positively related to knowledge acquisition, as evidenced by higher scores on post-test [5] [6].

Two studies were conducted on already working nurses and midwives; Simulation has been shown to increase the understanding of procedures than lecture methods when used to educate already practising healthcare's providers [7] [8].

Two studies [9] [10] reviewed looked at resident Anesthesiology residents and Obstetrics/gynaecological residents and found Simulation training to be superior to traditional lecture alone for teaching crucial skills [11] [12]. The last population used in this literature review was medical students with the result from post-test to delayed post-test, showing the simulation group demonstrated significant improvement, while the lecture group scores remained similar [13]. 


\subsubsection{Strategy (S)}

All the included studies recommended simulation as a viable substitute for traditional lecture and justified the large capital expenditures associated with its implementation and use. The project supported the author's Project question in the use of simulation for teaching to increase the knowledge level of clinical competence to bridge the theory-practice gap in new graduates. Overall, simulation was recognized as a safe way to learn, and most students enjoyed participating in it.

\subsubsection{Comparison (C)}

The review studies compared simulation against a traditional, didactic lecture teaching strategy. Half of the studies reviewed compared simulation with lecture and concluded that lecture alone as a teaching strategy is less effective in acquiring clinical competence [6] [8] [11] [12] [13]. Jawaher et al. (2015) [9], recommended the combination of lecture with simulation to increase student performance and satisfaction.

\subsubsection{Outcome (0)}

The review and synthesis of literature supported the use of simulation for assisting nursing students to feel more confident in performing clinical work. The use of simulation supported the curriculum and enhanced the knowledge level and learning of each student as the faculty continued to use simulation throughout the nursing courses [5] [6] [11]. Most [6] of the studies used pre-test and posttest to measure clinical competence [6] [7] [8] [10] [12] [13]. Ross, (2016) [6] uses a practical rating scale to measure clinical competency in intramuscular injection after simulation.

\section{Discussion}

The studies included in the literature review consisted of two systemic reviews (level II), five randomized controlled trials (level I) and three quasi-experimental studies (level II). The studies were conducted in multiple countries and included nursing and other health professionals from a variety of specialties, which supports the ability to generalize the findings and use these methods when teaching course content to different student populations. Summarized below are the similarities and differences in the literature and research methods. Gaps in the literature are addressed along with how the literature will influence the execution of the teaching strategy project.

About similarities and differences in the literature, of the 520 articles returned for the literature search, 401 (77.1\%) compared simulation with other active teaching strategies, $11(2.1 \%)$ identified using simulation combined with lecture, 98 (18.8\%) identified using simulation pretest and posttest and 10 (1.9\%) identified using simulation compared with a lecture. The search results indicate that research is abundant currently exists for simulation.

Simulation training is superior to traditional lectures for teaching crucial clinical skills, also simulation-based education is an effective educational strategy to 
teach nurses clinical skills and simulation has also been shown to increase the understanding of healthcare's complexities and to promote professional communication [6]-[13].

Additionally, simulation and lecture studies in the body of evidence identified to support that nursing education benefits from lectures to be combined with simulation to increase student performance and satisfaction most significantly [8]. In four of the studies, simulation was implemented in a single intervention over some time with pretest and posttest used to measure the effectiveness in acquiring clinical competency [6] [7] [9] [10].

Simulation leads the way for the future of nursing knowledge and performance of students to demonstrate knowledge, build self-confidence, develop critical thinking skills, develop psychomotor skills, and prepare for the transition to become a competent nurse. Technology is now and will continue in healthcare. Using simulation technology will grow and enhance the nurses of tomorrow to provide safe and high-quality care for the populations into the next generations. The current focus in health care on patient safety and evidence-based education requires nurse educators to utilize the most effective evidence-based teaching strategies when preparing future nurses.

\section{Limitation}

A significant limitation of the literature in the current project was the small sample sizes ranging from 20 [11] [13] to 98 [10] participants in the single research studies. The statistical significance of research findings could be further validated if the studies were to be repeated with the same conditions but using larger sample sizes. Additionally, the use of various instruments for measuring clinical competency in each of the studies may limit the ability to replicate their findings while the validity of each instrument for measuring clinical competency in this population varied.

Quantitative assessment data from nine studies provided supportive evidence that simulation was associated with significant improvement in the development of clinical competence-related skills in nursing students compared to a lecturebased teaching method [5] [6] [8]-[13]. One study though agreeing with the other studies but further suggested that the combination of lecture with simulation increases student performance and satisfaction. The evidence gathered in the project have adequately answered the PSCOT question positively.

\section{Conclusions}

The project, along with relevant nursing education literature, answered the PSCOT question which supports simulation increased clinical competency in new graduate nurses as compared with a lecture. Simulation is an effective teaching modality for psychomotor skills and has the potential for enhanced skill transfer to the clinical setting.

Due to this dearth of empirical evidence supporting simulation in psychomo- 
tor skill attainment and skill transference in the nursing literature, the underpowered findings of the current project, and the call from the National League of Nurses (NLN) for research to link education and practice (2012), is important that future nursing education research efforts explore the transfer of skills to clinical practice following simulation. Replication of the current studies in the project with larger sample size, as well as with varied psychomotor skills, would greatly enrich the body of knowledge in nursing education and provide the needed support for evidence-based education using simulation. Future research should also investigate whether knowledge gained from the simulation is more persistent over time than that from traditional teaching methods.

\section{Conflicts of Interest}

The authors declare no conflicts of interest regarding the publication of this paper.

\section{References}

[1] Bell, S.A., Rominski, S., Bam, V. and Donkor, L.J. (2013) Analysis of Nursing Education in Ghana: Priorities for Scaling-Up the Nursing Workforce. Nursing \& Health Sciences, 15, 244-249. https://doi.org/10.1111/nhs.12026

[2] Christmals, C., Gross, J., Aziato, L. and Armstrong, J.S. (2018) The State of Nursing Research in Ghana: An Integrative Literature Review. SAGE Open Nursing, 4.

https://doi.org/10.1177/2377960818783820

[3] Adu-Gyamfi, S. and Brenya, E. (2016) Nursing in Ghana: A Search for Florence Nightingale in an African City. International Scholarly Research Notices, 2016, Article ID: 9754845 . https://doi.org/10.1155/2016/9754845

[4] Hassanien, M.A. (2017) Strategic Planning in Higher Education, a Need for Innovative Model. Journal of Education, Society and Behavioural Science, 23, 1-11. https://doi.org/10.9734/JESBS/2017/37428

[5] Aebersold, M. (2018) Simulation-Based Learning: No Longer a Novelty in Undergraduate Education. OJIN: The Online Journal of Issues in Nursing, 23, 1. https://doi.org/10.3912/OJIN.Vol23No02PPT39

[6] Flood, L.S. and Higbie, J. (2016) A Comparative Assessment of Nursing Students' Cognitive Knowledge of Blood Transfusion Using Lecture and Simulation. Nurse Education Practice, 16, 8-13. https://doi.org/10.1016/j.nepr.2015.05.008

[7] Bodine, J.L. and Miller, S. (2017) A Comparison of Lecture versus Lecture plus Simulation. Journal of Hospice \& Palliative Nursing, 19, 34-40. https://doi.org/10.1097/NJH.0000000000000302

[8] Esfahani, M.S., Taleghani, F., Noroozi, M. and Tabatabaeian, M. (2018) An Educational Intervention on Based Information, Motivation and Behavior Skills Model and Predicting Breast Self-Examination. Journal of Preventive Medicine and $\mathrm{Hy}$ giene, 59, E277-E281.

[9] Jawaher Bin, J. and Ruland, J.P. (2015) A Critical Review of Simulation-Based on Nursing Education Research: 2004-2011. International Journal of Nursing Education, 7, 135-139. https://doi.org/10.5958/0974-9357.2015.00151.8

[10] Stayt, L.C., Merriman, C., Ricketts, B., Morton, S. and Simpson, T. (2015) Recognizing and Managing a Deteriorating Patient: A Randomized Controlled Trial In- 
vestigating the Effectiveness of Clinical Simulation in Improving Clinical Performance in Undergraduate Nursing Students. Journal of Advanced Nursing, 71, 25632574. https://doi.org/10.1111/jan.12722

[11] Ramsingh, D., Rinehart, J., Kain, Z., Strom, S., Canales, C., Alexander, B., Capatina, A., Ma, M., Van Le, K. and Cannesson, M. (2014) Impact Assessment of Perioperative Point-of-Care Ultrasound Training on Anesthesiology Residents. Anesthesiology, 123, 670-682. https://doi.org/10.1097/ALN.0000000000000776

[12] Fisher, N., Bernstein, P.S., Satin, A., Pandanani, S., Heo, H., Merkatz, I.R. and Goffman, D. (2010) Resident Training for Eclampsia and Magnesium Toxicity Management: Simulation or Traditional Lecture? American Journal of Obstetric Gynecology, 203, 379.e1-5. https://doi.org/10.1016/j.ajog.2010.06.010

[13] Alluri, R.K., Tsing, P., Lee, E. and Napolitano, J. (2015) A Randomized Controlled Trial of High-Fidelity Simulation versus Lecture-Based Education in Preclinical Medical Students. Medical Teacher, 38, 404-409.

https://doi.org/10.3109/0142159X.2015.1031734

\section{Abbreviations}

CINAHL: Cumulative Index to Nursing and Allied Health Literature

EBP: Evidence Based Project

NLN: National League of Nursing

PubMed: Publisher MEDLINE

PSCOT: In the registered general nursing (RGN) students (P) how does simulation (S) compared with lecture $(\mathrm{C})$ affect clinical competency? $(\mathrm{O})$ at the completion of the program $(\mathrm{T})$ 